\title{
Efeitos do chumbo sobre o cérebro em desenvolvimento
}

\section{Lead effects on the developing brain}

\author{
Carolina A. R. Funayama
}

\begin{abstract}
RESUMO
No presente trabalho procedeu-se à revisão de literatura dos efeitos do chumbo sobre o cérebro, com enfoque no desenvolvimento infantil. O chumbo pode permanecer no organismo durante toda a vida do indivíduo, sendo difícil a sua remoção. Não há nível estável seguro, considerando sua passagem lenta dos ossos para o sangue, com aceleração em condições como na gravidez. Há evidências de que o cérebro imaturo é um dos mais importantes alvos, e as pesquisas apontam para consequências mais tardias, em processos cerebrodegenerativos no adulto. Na infância, mesmo níveis sanguineos inferiores a 10 ?g/dL podem afetar a cognição. Estudos clínicos registram seus efeitos tóxicos na criança em idade escolar e na adolescência trazendo prejuízos motores, sensoriais, adaptativos, cognitivos e comportamentais, destacando-se a delinquência. Conclui-se, pela gravidade dos efeitos e dificuldade de tratamento, que o enfoque na orientação populacional sobre as medidas preventivas da exposição ao chumbo é prioritário.
\end{abstract}

Palavras-chave: Sistema Nervoso. Chumbo. Criança. Desenvolvimento Infantil. Revisão.

$\mathrm{O}$ efeitos danosos do chumbo sobre o sistema nervoso central vêm recebendo cada vez mais atenção de pesquisadores nas últimas décadas, evidenciando seus mecanismos nos processos de aprendizagem e memória, nas relações com o sistema de estresse, liberação de neurotransmissores e plasticidade sináptica, entre outros1. Os astrócitos são o principal tipo de célula nervosa onde o chumbo se deposita, cerca de 24 vezes mais em relação aos neurônios. $^{2,3}$

Quanto aos seus efeitos sobre os sítios da memória no hipocampo, foi observado em estudo in vitro ${ }^{4}$ que a Proteína-78 regulada pela glicose (GRP78), uma das proteínas de choque térmico envolvidas na produção de antígenos, encontra-se reduzida em células hipocampais em cultivo com presença de chumbo. Segundo esses autores, a GRP78 está envolvida na secreção da interleucina 6 (IL6) pelos astrócitos, e o achado de sua deficiência no hipocampo leva à hipótese de que a suscetibilidade ao chumbo em período precoce do desenvolvimento poderá causar neurodegeneração na vida adulta. Outros estudos recentes ${ }^{5,6}$ apontam que uma kinase regulada por sinal extracelular (ERK 1/2), da mesma natureza da GRP78, está envolvida com o processo de potenciação de longa duração (LTP - long term potentiation). A plasticidade sináptica do giro denteado hipocampal foi testada por análise neurofisiológica, avaliando-se o efeito do quercetin, um potente antioxidante, durante a exposição crônica ao chumbo. ${ }^{7}$ Esses autores estudaram, entre
1. Docente. Departamento de Neurologia, Psiquiatria e Psicologia Médica. Faculdade de Medicina de Ribeirão Preto - USP.
Correspondência: Departamento de Neurologia, Psiquiatria e Psicologia Médica. Faculdade de Medicina de Ribeirão Preto - USP. 14048-900 - Ribeirão Preto, SP

Artigo recebido em 12/02/2009 Aprovado em 08/12/2009 
outros registros, o LTP, que mostrou-se significantemente maior no grupo de animais que recebeu quercetin, sugerindo o efeito protetor do antioxidante, e portanto, corroborando o efeito danoso do chumbo no sistema sináptico. Outro estudo deste mesmo grupo de pesquisa ${ }^{8}$ também aponta para a proteção dada pela associação de clioquinol, um agente quelante, quando associado à vitamina B12.

$\mathrm{Na}$ mesma direção da hipótese do dano progressivo, desta vez com oportunidade de verificar outras regiões cerebrais, a avaliação volumétrica por exame de ressonância magnética do encéfalo evidenciou que a exposição do sistema nervoso durante a infância está associada na fase adulta à redução de regiões específicas, principalmente porções do córtex pré-frontal e cingulado, que, além das funções executivas, são responsáveis por regulação do humor e capacidade de decisão, sendo tais achados mais pronunciados no gênero masculino. ${ }^{9}$ Nesse sentido, cabe a atenção especial ao agravamento ou mesmo aparecimento com o avançar da idade, de doenças mentais como distúrbios do humor e de sinais do transtorno do déficit de atenção e hiperatividade.

Quanto à relação do estresse materno com os efeitos do chumbo nos fetos, na presença dos dois, isto é, estresse mais a exposição ao chumbo, o impacto negativo sobre os sistemas mesolímbicos da dopamina e glutamato envolvidos em funções cognitivas é maior do que quando se testa em separado cada um (estresse ou chumbo) durante a prenhez, segundo estudo experimental. ${ }^{10}$

Focalizando pesquisas clínicas em humanos, deve ser salientado que estas enfrentam ainda desafios como a cinética do chumbo nos diversos órgãos e suas relações endocrinológicas, ${ }^{11}$ o método de dosagem de chumbo e como obter os melhores valores de toxicidade. ${ }^{12,13}$ Além disso, falta ainda definição do nível mínimo a ser considerado danoso, ${ }^{14}$ o que se torna mais complexo, frente aos achados de diferentes suscetibilidades genéticas. ${ }^{15,16}$

Tem ocorrido importante avanço na identificação dos períodos mais vulneráveis durante o desenvolvimento infantil. Crianças estão expostas a riscos de intoxicação por chumbo desde o período pré-natal: durante a gravidez e lactação, na mulher ocorre importante mobilização do chumbo da parte óssea para o sangue, ${ }^{17}$ aumentando, portanto, o risco para o bebê, tanto na fase pré-natal, não se sabe se embrionária, mas com certeza fetal e na de lactente, tendo sido demonstrada correlação entre níveis de chumbo no leite materno e no sangue do recém-nascido. ${ }^{18}$ Entretanto não há ainda diretrizes para orientação quanto ao nível de chumbo materno, seja no colostro ou sangue ou ósseo para definir a suspensão do aleitamento.

Os níveis de chumbo no sangue aumentam entre o nascimento e dois anos de idade, com um pico entre 18 e 24 meses, período no qual as crianças adquirem o hábito de levar objetos à boca. A prevalência em amostra populacional norte americana de níveis acima de $10 \mu \mathrm{g} / \mathrm{dL}$ na idade de um ano foi em torno de $17 \%$ e, retestados aos 24 meses a taxa foi de $29 \% .{ }^{14}$ Existem evidências de que, em crianças, o chumbo em excesso produz efeitos adversos em rins ${ }^{19}$ e sistema hematopoiético, ${ }^{20}$ interfere no peso ao nascimento, ${ }^{21}$ perímetro craniano ${ }^{22}$ e há controvérsias quanto ao crescimento; ${ }^{23}$ reduz a acuidade auditiva e equilíbrio, além de séria interferência nas funções cognitivas. ${ }^{24,25,26}$ Needleman et al $(1972)^{24}$ foi um dos pioneiros na luta para o reconhecimento dos efeitos danosos desse metal e apresentaram os primeiros estudos em crianças americanas. Em $1983^{25}$ observaram que mais de 3000 crianças frequentando primeira e segunda séries foram expostas a chumbo no passado, em análise da concentração de chumbo nos dentes, e que variáveis neuropsicológicas, nível intelectual, processamento auditivo central e escores em eletrencefalograma foram piores em crianças com os mais altos níveis de chumbo. Com as medidas para redução das taxas de chumbo na gasolina e nas tintas houve redução do número de crianças intoxicadas, mas os riscos das residências com pinturas antigas, anteriormente ao período de 1978 ainda representam séria ameaça para as crianças americanas. Estudos em diversas partes do mundo apontam para a relação entre níveis altos de chumbo no sangue e alteração no desenvolvimento infantil. Em $1990^{26}$ meta-análise de 24 estudos sobre relação entre níveis de chumbo e quociente de desenvolvimento ou inteligência infantil, aponta que plumbemia mesmo abaixo de $10 \mu \mathrm{g} / \mathrm{dL}$ pode causar danos. Tais achados têm sido corroborados por dados recentes. Em Cincinati ${ }^{27}$ registraram escores de inteligência reduzindo proporcionalmento ao aumento das taxas de chumbo, mesmo naquelas crianças com níveis abaixo de $10 \mu \mathrm{g} / \mathrm{dL}$. Entre 276 com 6 meses de idade houve redução de 11 pontos no quociente de desenvolvimento e redução de 5 pontos a cada $10 \mu \mathrm{g} / \mathrm{dL}$ a mais. Assim como nestes, em outros estudos tem sido observado que o chumbo não somente interfere na maturação de funções cognitivas já estabelecidas, mas também afeta aquelas em desenvolvimento no 
presente e ainda as que vão surgir, como a capacidade de organização, planejamento, senso crítico e de estabelecer limites, inibição, raciocínio numérico. ${ }^{28,29}$

Alguns estudos tem apontado relação entre níveis altos de chumbo na infância e criminalidade na juventude. ${ }^{30-33}$ Recentemente extensa pesquisa envolvendo dados forenses ${ }^{33}$ demonstra forte associação entre níveis elevados de chumbo em crianças no período pré-escolar e criminalidade de vários tipos, analisando as tendências internacionais das taxas de criminalidade ao longo de várias décadas desde 1940, evidenciando, por análise de regressão múltipla, a forte relação entre períodos críticos de elevado nível de exposição ao chumbo (pinturas em casas antigas e gasolina) e as maiores taxas de criminalidade, enquanto o contrário também ocorreu principalmente no período após as medidas de retirada do chumbo da gasolina.

Considerando-se as potenciais perdas neurológicas ao longo da vida, bem como as dificuldades no tratamento para remoção do chumbo do organismo, ${ }^{34}$ não resta dúvidas de que a medida mais importante é a prevenção. Nesse sentido, as principais fontes de contaminação por chumbo devem ser incluídas em anamnese clínica em nível primário de atendimento, como uma medida no sentido da vigilância desse risco, bem como na consulta pré-natal, lembrando que pode haver associação da concentração materna de chumbo com abortos. ${ }^{35} \mathrm{Na}$ vertente da saúde púbica, vem ocorrendo a participação efetiva do governo na elaboração de leis que estabelecem os limites para o uso de chumbo nos diversos setores de fabricação, bem como sobre os cuidados com armazenamento e descarte dos produtos contaminantes. Entretanto, as experiências nacionais em regiões contaminadas ${ }^{36-39}$ indicam a necessidade de capacitação de equipes de saúde e controle ambiental específicas na missão de ampliar o alcance na identificação de áreas de risco, definição de programas armazenamento, manejo e remoção de agentes contaminantes, incluindo medidas efetivas de prevenção junto à população.

\begin{abstract}
This work is a review of the lead effects on the brain, focusing on child development. Lead can remain in the body throughout the life, being difficulty its removal, and there is no safe stable level, considering its slow passage of the bones for blood, but acceleration in moments such as pregnancy. There is evidence that the immature brain is one of the most important targets. Surveys point to later consequence in adult life, as the degenerative brain disease. In the childhood, even blood levels under 10 ?g/dL may affect cognition. Clinical studies record their toxic effects in childhood and adolescence, bringing motor, sensory, adaptive, cognitive and behavioral losses, highlighting the crime itself. It is due to the severe effects on the developing nervous system and treatment difficulties, that the priority is the population guidance on the preventive measures of exposure to lead.
\end{abstract}

Keywords: Nervous System. Lead. Child. Child Development. Review.

\section{Referências Bibliográficas}

1. White LD, Cory-Slechta DA, Gilbert ME, Tiffany-Castiglioni E, Zawia $\mathrm{NH}$, Virgolini $\mathrm{M}$ et al. New and evolving concepts in the neurotoxicology of lead. Toxicol Appl Pharmacol. 2007; 225: 1-27.

2. Sierra EM, Tiffany-Castiglioni E. Reduction of glutamine synthetase activity in astroglia exposed in culture to low levels of inorganic lead. Toxicology. 1991; 65:295-304.

3. Tiffany-Castiglioni E. Cell culture models for lead. Neurotoxicology. 1993; 14:513-36.

4. Qian Y, Zheng Y, Weber D, Tiffany-Castiglioni E. A 78-kDa glucose-regulated protein is involved in the decrease of interleukin- 6 secretion by lead treatment from astrocytes. Am J Physiol Cell Physiol. 2007; 293:897-905.

5. Adams JP, Sweatt JD. Molecular psychology: roles for the ERK MAP. Ann Rev Pharmacol Toxicol. 2002; 42:135-63.
6. Zhang Y, Ye L, Wang B, Cao S, Sun L. Effect of lead on ERK activity and the protective function of bFGF in rat primary culture astroglia. J Zhejiang Univ Sci. B 2007; 8:422-7.

7. Hu P, Wang M, Chen WH, Liu J, Chen L, Yin ST et al. Quercetin relieves chronic lead exposure-induced impairment of synaptic plasticity in rat dentate gyrus in vivo. Naunyn Schmiedebergs Arch Pharmacol. 2008; 378:43-51.

8. Chen WH, Wang M, Yu SS, Su L, Zhu DM, She JQ et al. Clioquinol and vitamin B12 (cobalamin) synergistically rescue the lead-induced impairments of synaptic plasticity in hippocampal dentate gyrus area of the anesthetized rats in vivo. Neuroscience. 2007; 147:853-64.

9. Cecil KM, Brubaker CJ, Adler CM, Dietrich KN, Altaye M, Egelhoff JC et al. Decreased brain volume in adults with childhood lead exposure. PLoS Med. 2008; 27: 112.

10. Virgolini MB, Chen K, Weston DD, Bauter MR, Cory-Slechta DA. Interactions of chronic lead exposure and intermittent 
stress: consequences for brain catecholamine systems and associated behaviors and HPA axis function. Toxicol Sci. 2005; 87:469-82.

11. Moreira FR, Moreira JC. A cinética do chumbo no organismo humano e sua importância para a saúde. Ciênc Saúde Coletiva. 2004; 9:167-81

12. Fernando Barbosa Jr F, Tanus-Santos JE, Gerlach RF, Parsons PJ.A critical review of biomarkers used for monitoring human exposure to lead: advantages, limitations and future needs. Ciênc Saúde Coletiva. 2006; 11: 229-41.

13. Guerra CS. Utilização de Plateau na concentração de chumbo no esmalte decíduo permite identificar crianças contaminadas.. [Dissertação] Ribeirão Preto. Faculdade de Farmácia e Odontologia - USP; 2007.

14. Bernard SM. Should the Centers for Disease Control and Prevention's Childhood lead poisoning intervention level be lowered? Am J Public Health. 2003; 93:1253-60.

15. Montenegro MF, Barbosa F Jr, Tanus-Santos JE. Assessment of How Pregnancy modifies plasma lead and plasma/whole blood lead ratio in ALAD 1-1 genotype women. Basic Clin Pharmacol Toxicol. 2008; 102:347-51.

16. Montenegro MF, Barbosa F Jr, Sandrim VC, Gerlach RF, TanusSantos JE. A polymorphism in the delta-aminolevulinic acid dehydratase gene modifies plasma/whole blood lead ratio. Arch Toxicol. 2006; 80:394-8.

17. Téllez-Rojo MM, Hernández-Avila $M$, Lamadrid-Figueroa $H$, Smith D, Hernández-Cadena L et al. Impact of bone lead and bone resorption on plasma and whole blood lead levels during pregnancy. Am J Epidemiol. 2004; 160:668-78.

18. Ettinger AS, Téllez-Rojo MM, Amarasiriwardena C, GonzálezCossío T, Peterson KE, Aro A et al. Levels of lead in breast milk and their relation to maternal blood and bone lead levels at one month postpartum. Environ Health Perspect. 2004; 112:926-31.

19. Burbure C, Buchet JP, Leroyer A, Nisse C, Haguenoer J-M, Mutti $A$ et al. Renal and neurologic effects of cadmium, lead, mercury, and arsenic in children: evidence of early effects and multiple interactions at environmental exposure levels. Environ Health Perspect. 2006; 114:584-90.

20. Papanikolaou NC, Hatzidaki EG, Belivanis S, Tzanakakis GN, Tsatsakis AM Lead toxicity update. A brief review. Med Sci Monit. 2005; 11:329-36.

21. González-Cossío T, Peterson KE, Sanín LH, Fishbein E, Palazuelos E, Aro A, Hernández-Avila M, Hu H. Decrease in birth weight in relation to maternal bone-lead burden. Pediatrics. 1997; 100:856-62.

22. Rothenberg SJ, Schnaas L, Perroni E, Hernández RM, Martínez S, Hernández C. Pre- and postnatal lead effect on head circumference: a case for critical periods. Neurotoxicol Teratol. 1999; 21:1-11.

23. Mahram M, Mousavinasab N, Dinmohammadi H, Soroush S, Sarkhosh F. Effect of living in lead mining area on growth. Indian J Pediatr. 2007; 74:555-9.

24. Needleman HL, Tuncay OC, Shapiro IM. Lead Levels in deciduos teeth of urban and suburban American children. Nature. 1972; 235:11-112.
25. Needleman HL. Lead at low dose and the behavior of children. Acta Psychiatr Scand., Suppl 1983; 303:26-37.

26. Needleman HL, Gatsonis CA. Low-level lead exposure and the IQ of children: a meta-analysis of modern studies. JAMA 1990; 263:673-8.

27. Lanphear BP, Hornung R, Khoury J, Yolton K, Baghurst P, Bellinger DC, et al. Low-level environmental lead exposure and children's intellectual function: an international pooled analysis. Environ Health Perspect. 2005; 113:894-99.

28. Wang CL, Chuang HY, Ho CK, Yang CY, Tsai JL, Wu TS, et al. Relationship between blood lead concentrations and learning achievement among primary school children in Taiwan. Environ Res. 2002; 89:12-8.

29. Johann M Vega-Dienstmaier JM, Salinas-Piélago JE, GutiérrezCampos MR, Mandamiento-Ayquipa RD, Yara-Hokama MC, Ponce-Canchihuamán J Castro-Morales J. Lead levels and cognitive abilities in Peruvian children. Rev Bras Psiquiatr. 2006; 28:33-9.

30. Denno DW. Biology and violence. New York: Cambridge University Press; 1990.

31. Dietrich K, Ris M, Succop P, Berger O, Bornschein R,. Early exposure to lead and juvenile delinquency. Neurotoxicol Teratol. 2001; 23:511-8.

32. Needleman HL, Riess JA, Tobin MJ, Biesecker GE, Greenhouse JB. Bone lead levels and delinquent behavior. JAMA. 1996; 275:363-9.

33. Nevin R. Understanding international crime trends: the legacy of preschool lead exposure. Environ Res. 2007; 104:31536.

34. Treatment of Lead-Exposed Children (TLC)-Trial Group. Safety and efficacy of succimer in toddlers with blood lead levels 20-44 ug/dL. Pediatr Res. 2000; 48:593-9.

35. Lamadrid-Figueroa $H$, Téllez-Rojo MM, Hernández-Avila $M$, Trejo-Valdivia B, Solano-González M, Mercado-Garcia A et al. Association between the plasma/whole blood lead ratio and history of spontaneous abortion: a nested cross-sectional study. BMC Pregnancy Childbirth. 2007; 7:22.

36. Carvalho FM, Silvany Neto AM, Lima MEC, Tavares TM, Azaro MGA, Quaglia GMC. Chumbo e cádmio no sangue e estado nutricional de crianças, Bahia, Brasil. Rev Saúde Pública. (São Paulo) 1987; 21:44-50.

37. Santos Filho E, Souza e Silva R de, Barretto HHC, Inomata ONK, Lemes VRR, Sakuma AM, Scorsafava MA. Concentrações sanguíneas de metais pesados e praguicidas organoclorados em crianças de 1 a 10 anos. Rev Saúde Pública. (São Paulo)1993; 27:59-67.

38. Paoliello MM, De Capitán EM, da Cunha FG, Matsuo T, Carvalho Mde F, Sakuma A, Figueiredo BR. . Exposure of children to lead and cadmium from a mining area of Brazil. Environ Res. 2002; 88:120-8.

39. Padula NAMR, Abreu MH, Myiazaki LCY, yamita NE, Grupo de estudo da intoxicação por chumbo em crianças de BauruSP. Intoxicação por chumbo e saúde infantil: ações intersetoriais para o enfrentamento da questão. Cad. Saúde Pública. 2006 ; 22:163-71. 